\title{
Tecnura
}

\section{Desarrollo y fabricación de un microsensor de gas de baja potencia para la detección de amoniaco a bajas concentraciones}

\section{Development and manufacture of a low power gas microsensor for the detection of ammonia at low concentrations}

\author{
Daniel Rodríguez ${ }^{1}$, Juan Bonaparte ${ }^{2}$, Norberto Boggio ${ }^{3}$, Alejandro Fasciszewski ${ }^{4}$
}

Fecha de recepción: 3 de octubre de 2018

Fecha de aceptación: 15 de marzo de 2019

Cómo citar: Rodríguez, D., Bonaparte, J., Boggio, N.G. y Fasciszewski, A. (2019). Desarrollo y fabricación de un microsensor de gas de baja potencia para la detección de amoniaco a bajas concentraciones. Tecnura, 23(61), 23-30. doi: https://doi.org/10.14483/22487638.15353

\section{Resumen}

Contexto: En este trabajo se presenta el desarrollo y microfabricación de un sensor de gas cuyo funcionamiento se basa en las variaciones de su conductividad eléctrica en presencia de determinados gases. Para utilizar estos sensores en equipos portátiles, como monitores de gases, se requiere que la potencia utilizada para mantener la película sensible a la temperatura de funcionamiento sea muy baja. Los resultados de este desarrollo permiten contar además con una plataforma para la microfabricación de sensores de gas, sobre la que se pueda incorporar diversos tipos de películas sensoras.

Metodología: El microsensor desarrollado está constituido por una película delgada de $\mathrm{SnO}_{2}$ depositada sobre un sustrato de silicio micromaquinado. El diseño propuesto se realizó mediante simulaciones de los comportamientos mecánico y térmico. El procedimiento incluyó operaciones de microfabricación.
Posteriormente se realizó la caracterización eléctrica y se ensayó la sensibilidad frente a amoniaco gaseoso. Resultados: La microfabricación se llevó a cabo de forma satisfactoria (no se detallan todos los pasos en el presente trabajo), y se logró una alta tasa de sensores bien conformados por oblea. La caracterización del sensor frente a diferentes concentraciones de amoniaco gaseoso en aire dio como resultado una respuesta lineal para concentraciones entre 6 ppm a 50 ppm , donde este último es el límite permisible de exposición .

Conclusiones: Se desarrolló un proceso para fabricar un microsensor de gas de $\mathrm{SnO}_{2}$ de bajo consumo $(50 \mathrm{~mW})$. Se llevó a cabo la fabricación de los microsensores de manera satisfactoria, partiendo de simulaciones previas. En relación a trabajos futuros, la plataforma micromaquinada desarrollada, con el calefactor incorporado, permitirá utilizar distintas películas sensoras.

1 Licenciado en Física, doctor en Ciencia y Tecnología mención Física, investigador Departamento de Micro y Nanotecnología, Comisión Nacional de Energía Atómica. Buenos Aires, Argentina. Contacto: drodrig@tandar.cnea.gov.ar ORCID: https://orcid.org/0000-0001-6828-4132

2 Ingeniero electrónico, Departamento de Micro y Nanotecnología, jefe de división Micro y Nanofabricación, Departamento de Micro y Nanotecnología, Comisión Nacional de Energía Atómica. Buenos Aires, Argentina. Contacto: bonaparte@cnea.gov.ar ORCID: https://orcid. org/0000-0001-7209-6441

3 Licenciado en Química, doctor en Ingeniería de Microsistemas, investigador del Instituto de Nanociencia y Nanotecnología (INN-Conicet). Buenos Aires, Argentina. Contacto: boggio@cnea.gov.ar ORCID: https://orcid.org/0000-0002-5421-5598

4 Magíster en Ingeniería Elctrónica, ingeniero electrónico, director del Departamento de Micro y Nanotecnología, Comisión Nacional de Energía Atómica. Buenos Aires, Argentina. Contacto: afascisz@cnea.gov.ar ORCID: https://orcid.org/0000-0003-3704-3066 
Palabras clave: sensor de gas, películas delgadas, monitoreo ambiental.

\section{ABSTRACT}

Objective: This paper presents the development and manufacture of a gas microsensor whose operation is based on variations in electrical conductivity in the presence of certain gases. To use these sensors in portable equipment such as gas monitors, the power used to keep the film sensitive to the operating temperature is required to be very low. The results of this development provide with a platform for the manufacture of gas microsensors that allow different types of sensor films can be incorporated.

Methodology: The developed microsensor consists of a thin fil of $\mathrm{SnO}_{2}$ deposited on a micro-machined silicon substrate. The proposed design was carried out by simulations of mechanical and thermal behavior. The procedure included microfabrication operations, and then, the electrical characterization was performed and the sensitivity to gaseous ammonia was tested.

Results: Microfabrication was carried out satisfactorily (although this work does not detail all the steps of the process), and a high rate of well-formed sensors per sheet was achieved. The behavior of the sensor in the presence of different concentrations of gaseous ammonia in air was characterized and resulted in a linear response for concentrations between $6 \mathrm{ppm}$ to $50 \mathrm{ppm}$, and the latter is the permissible limit of exposure.

Conclusions: A low consumption (50 mW) $\mathrm{SnO}_{2}$ gas microsensor was successfully developed based on previous simulations. In relation to future work, the developed micromachined platform with the builtin heater will allow the use of different sensor films.

Keywords: gas sensor, thin films, environmental monitoring.

\section{INTRODUCCIÓN}

Los sensores de gas desempeñan un papel vital entre las aplicaciones tecnológicas más importantes en la cotidianidad actual. Los usos típicos de sensores de gas incluyen la detección de compuestos tóxicos y gases explosivos con fines de seguridad pública y doméstica, procesos industriales, el monitoreo de la contaminación ambiental y, más recientemente, en el diagnóstico de enfermedades de diverso tipo, la identificación del grado de maduración de frutos comestibles o la detección de pestes en los cultivos (Dey, 2018 ; Korotcenkov y Cho, 2017; Miller, Akbar y Morris, 2014; Mirzaei, Leonardi y Neri, 2016; Lamagna et al., 2008; Vorobioff et al., 2018).

Entre los diversos tipos de sensores de gas, los resistivos son los más atractivos debido a la facilidad de fabricación, operación simple, bajo costo de producción y miniaturización (Barsan, Koziej y Weimar, 2007; Korotcenkov, 2007; Franke, Koplin y Simon, 2006; Acosta Pérez, Suárez Castrillón y
Suárez Castrillón, 2016). Un sensor de gas resistivo típico contiene una película de detección activa, cuya conductividad es altamente sensible a las sustancias presentes en los ambientes circundantes. El primer dispositivo comercial de sensor de gas fue desarrollado en la década de 1960 utilizando óxido de metal como la capa de detección (Ihokura et al., 1994).

Desde entonces, la investigación en esta materia tuvo como objetivos mejorar la sensibilidad, la selectividad, la velocidad (respuesta y tasa de recuperación) y la estabilidad del sensor. El desarrollo en sensores de gas logró un progreso significativo impulsado por la nanociencia y la nanotecnología emergentes (Xu et al., 2018; Zhang, Liu, Neri y Pinna, 2016; Hoa, Duy, El-Safty y Hieu, 2015). Por otra parte, las crecientes necesidades de dispositivos pequeños requieren de la miniaturización de los sistemas de análisis. Esta ofrece beneficios no solamente económicos (la fabricación en serie cuesta menos), sino también la reducción de tamaños de muestra para análisis, portabilidad y consumo de reactivos. 
La sensibilidad del sensor $(S)$, definida como $R_{a} /$ $R_{g}$ ó $R_{g} / R_{a}$-donde $R_{a}$ y $R_{g}$ son resistencia eléctrica en aire y gas, respectivamente-, puede ser altamente dependiente del tamaño de grano (D) del óxido metálico, cuando el tamaño del grano es comparable a $2 \mathrm{~L}$-donde $\mathrm{L}$ es el espesor de la región de vaciamiento de electrones-. Cuando D es mucho mayor que $2 \mathrm{~L}$, solo el borde de grano está sujeto a la formación de la región de vaciamiento de electrones, lo que significa que el efecto de detección en superficie no afecta mucho a la resistencia del sensor (R). Para $\mathrm{D} \leq 2 \mathrm{~L}$, la mayor parte del grano o todo el grano se agota de electrones. En este caso, la resistencia del sensor es muy sensible para la detección, resultando así que la sensibilidad es altamente dependiente del tamaño del grano (Yamazoe, 1991). Junto con el tamaño de partícula, otras características microestructurales de los semiconductores de óxidos metálicos, como la morfología y la porosidad, también tienen influencia sobre la sensibilidad y la velocidad de respuesta del sensor, debido al ordenamiento de los átomos en ciertas caras del cristal, el aumento del área superficial y una mejor difusión del gas (Wagner et al., 2013; Lee, 2009).

Otro requerimiento importante de los sensores de gas es el funcionamiento de forma altamente precisa y selectiva con el fin de satisfacer las demandas de aplicaciones versátiles. Hasta el momento, se han desarrollado numerosas estrategias para mejorar la selectividad del sensor para un determinado gas, entre las que se cuentan la fabricación de nanocompuestos, la funcionalización de la superficie, el diseño de heteroestructuras (Vargas, 2018; Kaushik et al., 2015; Miller, Akbar y Morris, 2014; Alenez, Henley, Emerson y Silva, 2014) la utilización de zeolitas, etc.

Las características del sensado se relacionan con las reacciones, térmicamente activadas, entre las especies a ser detectadas, las partículas de la película sensora y las adsorbidas previamente. Estas reacciones conducen a una variación de la conductividad de la película, conducida al exterior por contactos y conductores eléctricos. Para optimizar la respuesta del dispositivo, en general se prefiere operar la película sensora a una temperatura controlada y uniforme elevada, normalmente de varios cientos de grados centígrados. A fin de mantener el consumo eléctrico dentro de niveles aceptables $(<100 \mathrm{~mW})$, es usual ubicar o integrar los calefactores y películas sensoras sobre una plataforma aislada térmicamente del sustrato portante del dispositivo.

En el presente trabajo se describe el desarrollo de un proceso de microfabricación para obtener un sensor de gas de película delgada con reducido consumo de potencia eléctrica.

\section{METODOLOGÍA}

El sensor propuesto se basa en una plataforma calefactora micromaquinada térmicamente aislada, dentro de la cual se integran los microcalefactores y los contactos eléctricos sobre los cuales descansa la película sensora. La plataforma se realizó mediante una película dieléctrica suspendida, sobre la cual se depositaron las termorresistencias que componen el calefactor y los contactos eléctricos de la película sensora. La suspensión de toda la estructura ofrece la aislación térmica necesaria.

El microsensor de gas que se desarrolló está constituido por una película delgada de $\mathrm{SnO}_{2}$ depositada sobre un sustrato de silicio micromaquinado. Esta película se encuentra calefaccionada térmicamente por un microcalefactor integrado en el microsensor para obtener una temperatura comprendida entre $200^{\circ} \mathrm{C}$ y $400^{\circ} \mathrm{C}$.

\section{Diseño y proceso de microfabricación propuesto}

Para el diseño del sensor se tuvieron en cuenta dos aspectos: lograr la mayor cantidad de sensores por oblea de silicio y que el proceso de microfabricación tuviera el menor número de pasos posibles.

Para analizar el diseño propuesto (figura 1) se realizaron dos tipos de simulaciones: 
- Mecánica: rigidez estructural, tamaños máximos y mínimos de estructuras suspendidas. Análisis de sensibilidad a las variaciones de los parámetros.

- Térmica: distribución de temperaturas y potencias eléctricas involucradas.

El proceso de fabricación está constituido por doce pasos que abarcan desde el depósito de la fotorresina hasta el encapsulado de los sensores individuales.

La totalidad de la estructura propuesta se pudo realizar con el equipamiento y personal disponible en la sala limpia del Departamento de Micro y Nanotecnología, Centro Atómico Constituyentes, CNEA, Argentina.

\section{RESULTADOS}

El proceso de microfabricación se llevó adelante de forma satisfactoria (no se detallan todos los pasos en el presente trabajo), logrando una alta tasa de sensores bien conformados por oblea (figura 2, a y b).

Posteriormente se cortó la oblea para obtener los sensores individuales (dicing). Esta etapa constituyó uno de los pasos críticos, dado que se produce una alta tasa de ruptura de las películas suspendidas.

Los sensores individuales bien conformados luego del dicing fueron montados en encapsulados (figura 3).

\section{Caracterización eléctrica}

Se realizó la caracterización eléctrica de los sensores utilizando una estación de prueba (figura 4) que permite medir los sensores individuales antes de realizar el encapsulado. En esta etapa se estudió la respuesta del calefactor midiendo la resistencia eléctrica frente a valores de tensión aplicada entre $0,1 \mathrm{~V}$ y $5 \mathrm{~V}$.

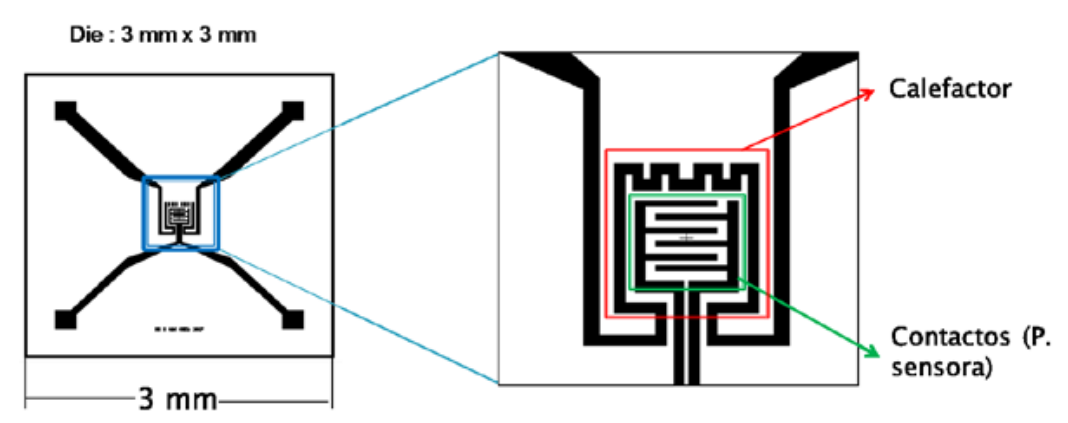

Figura 1. Esquema del sensor propuesto. En la ampliación de la parte central del sensor se puede observar el detalle del diseño.

Fuente: elaboración propia.

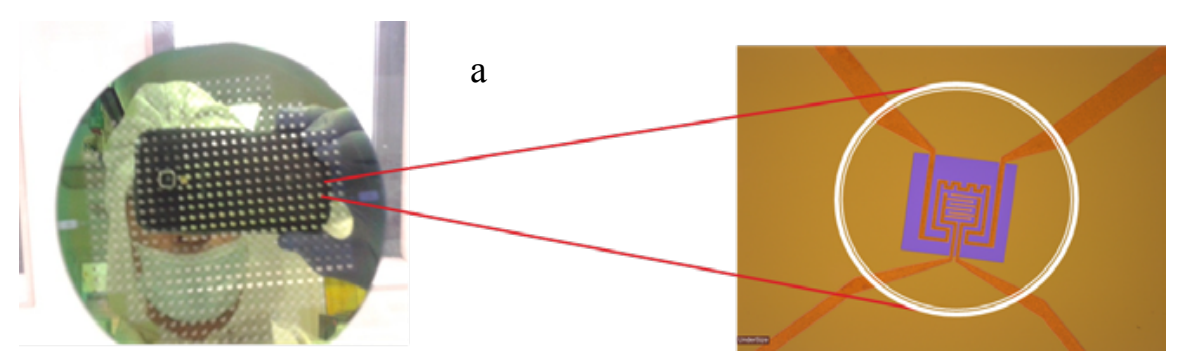

Figura 2. a) Oblea de silicio maquinada, b) fotografía de un sensor individual

Fuente: elaboración propia. 
A continuación se presentan una serie de mediciones para determinar la temperatura del sensor según la tensión aplicada al calefactor, sobre un sensor en particular:

De esta manera se puede regular el calentamiento de la película sensora al variar la tensión aplicada al calefactor.

Simultáneamente, durante las mediciones, en el microscopio se registraron las modificaciones superficiales que se producen durante el calentamiento al aplicarse las diferentes tensiones. La película sensora se encuentra suspendida sobre una membrana de nitruro de silicio (en color violáceo en las fotos), cuyo espesor es de alrededor de 250 $\mathrm{nm}$. Los cambios observados hacia una tonalidad más oscura al aumentar la tensión eléctrica, corresponden a deformaciones mecánicas de la membrana como resultado del calentamiento local, respecto de la periferia, más fría (figura 5).
Tabla 1. Valores de temperatura obtenidos de acuerdo con la tensión aplicada

\begin{tabular}{ccc}
\hline $\begin{array}{c}\text { Tensión } \\
\text { [voltios] }\end{array}$ & $\begin{array}{c}\text { Resistencia } \\
\text { [W] }\end{array}$ & $\begin{array}{c}\text { Temperatura } \\
{[\mathbf{o C}]}\end{array}$ \\
\hline 0,1 & 324 & $25($ T ambiente $)$ \\
\hline 1,0 & 342 & 30 \\
\hline 1,5 & 361 & 45 \\
\hline 2,0 & 382 & 62 \\
\hline 2,5 & 404 & 81 \\
\hline 3,0 & 427 & 101 \\
\hline 3,5 & 450 & 123 \\
\hline 4,0 & 472 & 143 \\
\hline 4,5 & 494 & 158 \\
\hline 5,0 & 516 & 180 \\
\hline
\end{tabular}

Fuente: elaboración propia.

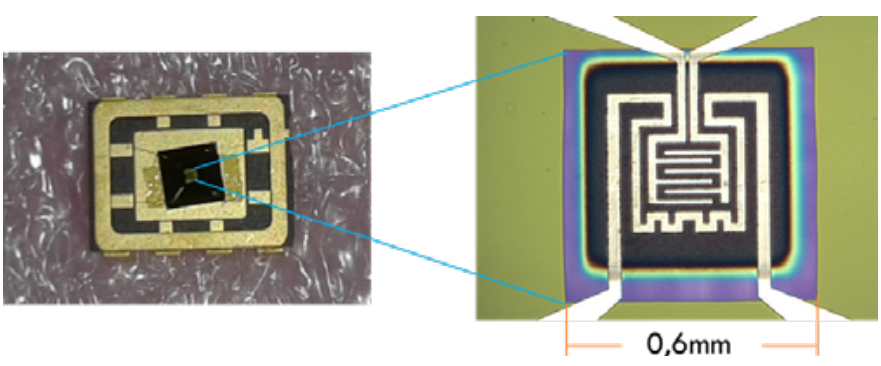

Figura 3. Sensor individual montado sobre un encapsulado

Fuente: elaboración propia.

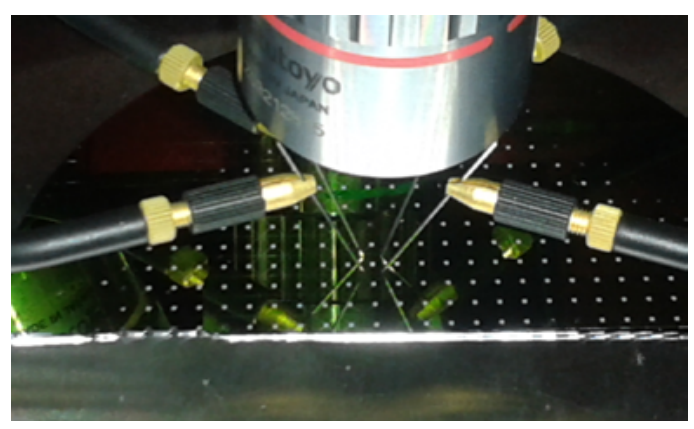

Figura 4. Se observan las puntas de prueba de la estación de prueba sobre los contactos eléctricos del microcalefactor y de la película sensible.

Fuente: elaboración propia. 


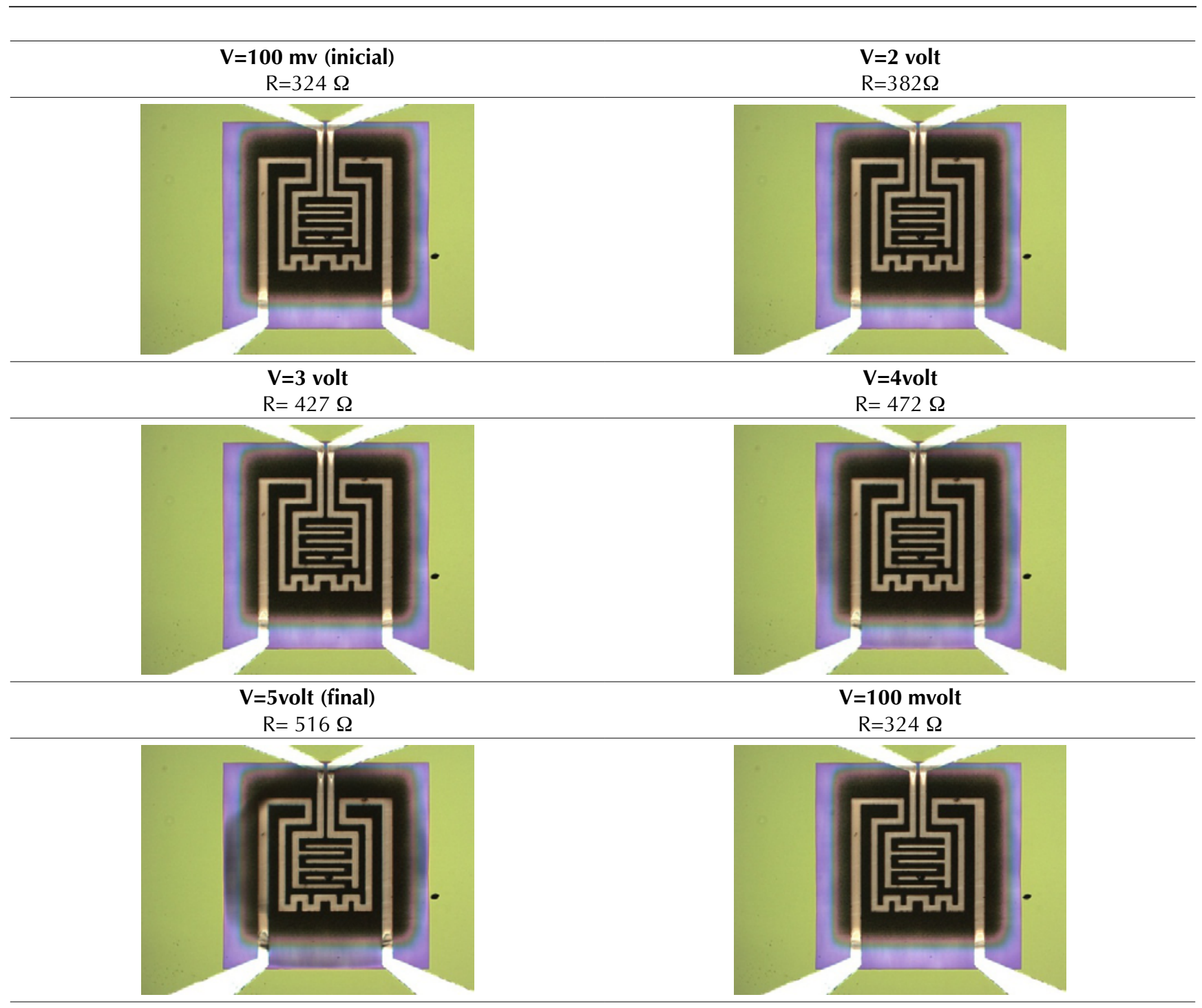

Figura 5. Secuencia del calentamiento de la película sensora

Nota: se observan los cambios superficiales en función de la tensión aplicada.

Fuente: elaboración propia

\section{Caracterización frente a gases}

Luego de ser encapsulados, se llevó a cabo la caracterización de los sensores, para lo cual se evaluó su respuesta en un banco de gases. Para ello se colocó cada sensor dentro de una cámara con un volumen aproximado de $75 \mathrm{~cm}^{3}$. Como gas portador, se inyectó aire sintético a un caudal controlado de 80 $\mathrm{cm}^{3} /$ min que a su vez constituye la línea de base.

A continuación se presentan los resultados correspondientes a un sensor ya encapsulado. Las mediciones fueron realizadas aplicando una tensión de $5 \mathrm{~V}$ al calefactor, obteniendo potencia disipada entre 44 y $49 \mathrm{~mW}$. La temperatura de operación de la película sensora en estas condiciones es de $180^{\circ} \mathrm{C}$ aproximadamente. Por otra parte la película sensora está conectada a un potencial 1 V. Se mide entonces la respuesta del sensor como la variación de la resistencia eléctrica en función del tiempo de respuesta. Los resultados se presentan en la figura 6. 


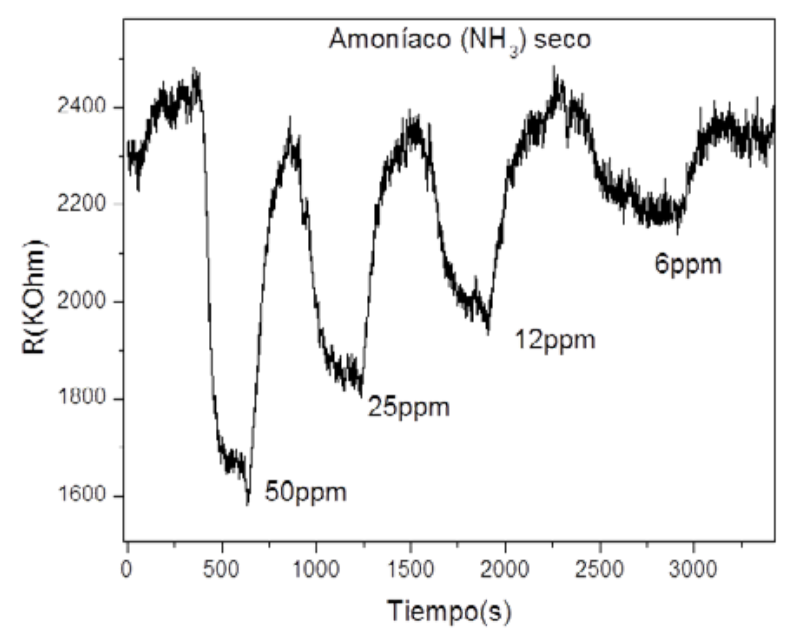

Figura 6. Respuesta del sensor vs. Tiempo de respuesta para diversas concentraciones de amoníaco gaseoso.

Nota: el límite de detección se estableció en 6 ppm.

Fuente: elaboración propia.

\section{CONCLUSIONES}

Se desarrolló un proceso para fabricar un microsensor de gas de $\mathrm{SnO}_{2}$ con un consumo del orden de $50 \mathrm{~mW}$.

De acuerdo con los objetivos propuestos, se llevó a cabo la fabricación de los microsensores de manera satisfactoria. Dentro de los pasos de fabricación la etapa crítica a mejorar es la de corte de la oblea en dices (sensores individuales), dada la alta tasa de ruptura de las membranas suspendidas.

En relación a trabajos futuros, la plataforma micromaquinada, con el calefactor incorporado, permite utilizar distintas películas sensoras, lo que ofrece una amplia variedad de aplicaciones, a requerimiento de instituciones públicas o privadas.

\section{REFERENCIAS}

Acosta Pérez, M.A., Suárez Castrillón, S.A. y Suárez Castrillón, A.M. (2016) Desarrollo y ensayo de un sensor químico de fibra óptica para la medición de concentraciones de dióxido de carbono. Tecnura, 20(50), 29-42. doi: http:// 10.14483/udistrital.jour. tecnura.2016.4.a02
Alenezi, M.R., Henley, S.J., Emerson, N.G. y Silva, S.R. (2014). Nanoscale, 6(1), 235-247.

Barsan, N., Koziej, D. y Weimar, U. (2007). Metal oxide-based gas sensor research: How to? Sens. \& Actuators B, 121(1), 18-35. doi: https://doi.org/10.1016/j.snb.2006.09.047

Dey A. (2018). Semiconductor metal oxide gas sensors: A review. Materials Science \& Engineering B, 229, 206-217. doi: https://doi.org/10.1016/j. mseb.2017.12.036

Franke, M.E., Koplin T.J. y Simon, U. (2006). Metal and metal oxide nanoparticles in chemiresistors. Does the nanoscale matter? Small, 2, 36-50. doi: https:// doi.org/10.1002/smll.200500261

Hoa, N.D., Duy, N.V., El-Safty, S.A. y Hieu, N.V. (2015). Meso-/Nanoporous Semiconducting Metal Oxides for Gas Sensor Applications. Journal of Nanomaterials, 2015, 1-12. doi: https://doi. org/10.1155/2015/972025

Ihokura, K. y Watson, J. (1994). The Stannic Oxide Gas Sensor Principles and Applications. Boca Raton, FL, EE. UU.: CRC Press.

Kaushik, A., Kumar, R., Arya, S.K., Nair, M., Malhotra, B. y Bhansali, S. (2015). Organic-Inorganic hybrid nanocomposite based gas sensors for environmental 
monitoring. Chemical Reviews, 115(11), 45714606. doi: https://doi.org/10.1021/cr400659h

Korotcenkov G., (2007) Metal oxides for solid state gas sensors: what determines our choice? Materials Science Engineering B, 139(1), 1-23. doi: https:// doi.org/10.1016/j.mseb.2007.01.044

Korotcenkov, G. y Cho, B.K. (2017). Metal oxide composites in conductometric gas sensors: Achievements and challenges. Sensors and Actuators B, 244, 182-210. doi: http://dx.doi.org/10.1016/j. snb.2016.12.117

Lamagna, A., Reich, S., Rodríguez, D., Boselli, A. y Cicerone, D. (2008) . The use of an electronic nose to characterize emissions from a highly polluted river. Sensors \& Actuators B, 131(1), 121-124. doi: https://doi.org/10.1016/j.snb.2007.12.026

Lee, J.H. (2009). Gas sensors using hierarchical and hoIlow oxide nanostructures: Overview. Sensors And Actuators B, 140(1), 319-336. doi: https://doi.org/10.1016/j.snb.2009.04.026

Miller, D.R., Akbar, S.A. y Morris, P.A. (2014). Nanoscale metal oxide-based heterojunctions for gas sensing: A review. Sensors \& Actuators B, 204, 250-272. doi: http://dx.doi.org/10.1016/j.snb.2014.07.074

Mirzaei, A., Leonardi, S.G. y Neri, G. (2016). Detection of hazardous volatile organic compounds (VOCs) by metal oxide nanostructures-based gas sensors: A review. Ceramics International 42, 15119-15141. doi: http://dx.doi.org/10.1016/j. ceramint.2016.06.145

Vargas, A.M.N. y Jaramillo, I. (2018). La industria de sensores en Colombia. Tecnura, 22(57), 44-54. doi: https://doi.org/10.14483/22487638.13518

Vorobioff, J., Adaro, M., Boggio, N., Magallanes, J., Boselli, A., Lamagna, A. y Rinaldi, C. (2018). Sensor Letters, 16(6), 410-414. doi: https://doi.org/10.1166/ sl.2018.3973

Wagner, T., Haffer, S., Weinberger, C., Klaus, D. y Tiemann, M. (2013). Mesoporous materials as gas sensors. Chemical Society Reviews, 42, 4036-4053. doi: 10.1039/C2CS35379B

Xu, K., Fu, C., Gao, Z., Wei, F., Ying, Y., Xu, C. y Fu, G. (2017). Nanomaterial-based gas sensors: A review. Instrumentation Science \& Technology, 46(2), 115145. doi: 10.1080/10739149.2017.1340896

Yamazoe, N. (1991). New approaches for improving semiconductor gas sensors. Sensors \& Actuators B, 5, 7-19. doi: https://doi. org/10.1016/0925-4005(91)80213-4

Zhang, J., Liu, X., Neri, G. y Pinna, N. (2016). Nanostructured Materials for Room- Temperature Gas Sensors. Advanced Materials, 28, 795-831. doi:10.1002/adma.201503825

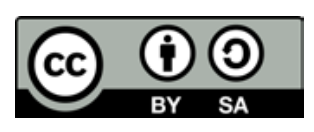

Tecnura • p-ISSN: 0123-921X • e-ISSN: 2248-7638 • Vol. 23 No. 61 • Julio - Septiembre de $2019 \bullet$ pp. 23-30 